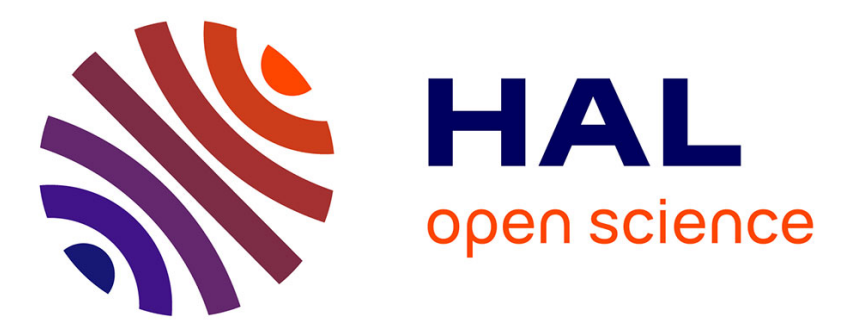

\title{
European research activities on charge state breeding related to radioactive ion beam facilities
}

\author{
T. Lamy, J. Angot, T. Thuillier
}

\section{To cite this version:}

T. Lamy, J. Angot, T. Thuillier. European research activities on charge state breeding related to radioactive ion beam facilities. 12th International Conference on Ion Sources (ICIS'07), 2007, Jeju, South Korea. pp.02A909, 10.1063/1.2819747 . in2p3-00142346

\section{HAL Id: in2p3-00142346 https://hal.in2p3.fr/in2p3-00142346}

Submitted on 29 Aug 2007

HAL is a multi-disciplinary open access archive for the deposit and dissemination of scientific research documents, whether they are published or not. The documents may come from teaching and research institutions in France or abroad, or from public or private research centers.
L'archive ouverte pluridisciplinaire HAL, est destinée au dépôt et à la diffusion de documents scientifiques de niveau recherche, publiés ou non, émanant des établissements d'enseignement et de recherche français ou étrangers, des laboratoires publics ou privés. 


\title{
European Research Activities on Charge State Breeding related to Radioactive Ion Beam Facilities ${ }^{a}$
}

\author{
T. Lamy ${ }^{1 *}$, J. Angot ${ }^{1}$, T. Thuillier ${ }^{1}$ \\ 1 Laboratoire de Physique Subatomique et de Cosmologie, Université Joseph Fourier \\ Grenoble 1, CNRS/IN2P3, Institut National Polytechnique de Grenoble, Grenoble, France \\ Fax: +33-(04)-7628-4143 E-mail address: lamy@lpsc.in2p3.fr
}

Abstract

European effort on charge breeders is mainly dedicated to present and future Radioactive Ion Beam facilities. The main projects are High Intensity and Energy-ISOLDE at CERN, SPIRAL2 at GANIL and EURISOL. Most of the experimental developments are funded by the European programs EURONS (EUROpean Nuclear Structure) and EURISOL (European Isotope Separation On-Line Radioactive Ion Beam Facility). Two ion source types (EBIS and ECRIS) have been adapted to accept the injection and the capture of an ion beam, in order to increase its charge with the highest efficiency within the shortest time. Both charge breeders have advantages and disadvantages with regard to their use in a Radioactive Ion Beam facility. The most important parameters studied are acceptance (in emittance and intensity) of the charge breeder, efficiency and charge breeding time of a specific $n+$ charge state, emittance of the extracted $n+$ beam. The charge breeder parameters are studied with different

\footnotetext{
${ }^{\text {a }}$ Invited paper, published as part of the Proceedings of the 12th International Conference on Ion Sources, Jeju, Korea, August 2007
} 
$1+$ ion sources dedicated to $1+$ radioactive ion beam production and the tuning procedure of the charge breeder as a beam line section of a specific accelerator is established and measured too.

\section{Introduction}

There are basically two kinds of Radioactive Ion Beam (RIB) facilities depending on the exotic nuclei production type: the Isotope Separator On Line (ISOL) where a high energy beam (primary beam) is directed on a thick hot target [1] and the in-flight method where the primary beam is thrown on a thin target [2]. There are numerous facilities presently working or under project in the world (a non exhaustive list is given Table 1). Efficient and fast charge breeding is a key point for present and future RIB facilities or for the physics of exotic nuclei [3], [4], [5]. The high charge states obtained for heavy elements either permit post acceleration by a cyclotron, or contribute to the cost reduction of linear accelerators. It has some applications too, for specific measurements on nuclear physics or astrophysics [6], [7]. There are two types of ion sources adapted in order to provide charge breeding of a $1+$ beam: the Electron Beam Ion Source (EBIS) and the Electron Cyclotron Resonance Ion Source (ECRIS). Another method can be used in order to enhance the charge of an ion beam at high energy: the stripping foil method, and finally one device is more dedicated to the Physics of the exotic nuclei themselves: the Electron Beam Ion Trap (EBIT) used as charge breeder.

Since the charge breeding concept came up, experimental studies were first performed in some laboratories in Europe in order to realize specific RIB facilities like PIAFE at Grenoble [8], [9] and REX-ISOLDE at CERN [10], [11]. The Nuclear Physics European Collaboration Committee (NUPECC) [12] makes recommendations to the European Science Foundation on the development and organization of nuclear science. The first priority defined by the NUPECC roadmap is the construction of the Facility for Antiproton and Ion Research (FAIR) at GSI, the second one is the construction of the European Isotope Separation On-Line RIB 
facility (EURISOL). These two facilities are mainly dedicated to nuclear structure physics and nuclear astrophysics with radioactive ion beams. The construction of these two facilities requires a tremendous $\mathrm{R} \& \mathrm{D}$ effort, the construction of intermediate-generation facilities like SPIRAL2, HIE-ISOLDE, SPES, is also recommended in order to perform research and applications with RIBs of the ISOL scheme and is the opportunity to progress in the scientific and technical challenges for the future. European charge breeding activities are a part of this R\&D effort, with the increasing integration of European research, they are nowadays mainly supported and funded by the European Commission.

\section{European Organization for charge breeding studies}

Charge breeding activities have the financial support from the European Community since 1999 in the frame of the 5th Framework Programme (FP5), this support has been re-granted within the 6th Framework Programme (FP6) under two structures: the European nuclear structure Integrated Infrastructure Initiative (EURONS) [13] and the European Isotope Separation On-Line RIB facility Design Study (EURISOL DS) [14].

\section{Charge breeding activities in EURONS}

The charge breeding activities, both for EBIS/T and ECRIS, in EURONS, are performed within the 'Joint Research Activity' 3 (JRA3). Five institutes participate to these activities (LMU München, LPSC Grenoble, CCLRC Daresbury laboratory, CERN, and MPI-K Heidelberg), the project coordination is performed by Dr. Oliver Kester (GSI). The purpose of the JRA is to optimize the charge breeding essentially acting on three parameters: the efficiency (including the charge breeding time), the beam purity and the $n+$ beam quality. The methods used to reach these objectives depend on the charge breeder type. 


\section{Charge breeding activities in EURISOL}

The purpose of the EURISOL Design Study is to prepare the construction of the nextgeneration ISOL RIB facility in Europe producing detailed engineering oriented studies and prototypes. It is divided in 12 tasks, charge breeding activities are grouped in Task 9 called 'Beam preparation', Dr Ari Jokinen (Jyväskylä University) is the task coordinator. Five institutions among the nine involved in this task focus their studies on charge breeding techniques (CERN, GSI, INFN-Bari, Ludwig-Maximilians-Universitaet Muenchen, Stockholm University -Manne Siegbahn Laboratory).

\section{Charge breeding activities related to specific facilities and experiments}

Beside the previous European programs, the SPIRAL2 facility has chosen the PHOENIX ECRIS charge breeder, LPSC Grenoble is responsible for the delivery of such a nuclearized charge breeder. Due to the high RIB's intensity expected $\left(\approx 10^{12} \mathrm{pps}\right)$, leading to a highly radioactive environment, the reliability of the technology of such a charge breeder has to be improved on specific identified weak points (the RF window for example). The charge breeder will be placed in a yellow area where human operation will be possible, but time limited, so the device will have to be mechanically optimized in order to limit the maintenance time. EBIS/T charge breeders are used or planned to be used on some facilities like REX-ISOLDE or the Isotope Science Facility (ISF) at NSCL-MSU [15]. Moreover they have a specific interest for physics on trapped exotic nuclei, like at HITRAP-GSI [16], or TITAN at TRIUMF [17], where the charge bred trapped ions permit to perform high precision mass-measurements on exotic nuclei. 


\section{Experimental charge breeding activities in Europe}

\section{EBIS/T charge breeding activities}

EBIS and EBIT principles have been extensively explained in Ref. [18], ions are radially and longitudinally confined by the space charge of an intense electron beam and an adequate electrostatic potential established by cylindrical electrodes. During the trapping, the ions are highly ionized by successive electron impacts. The main parameters which influence the efficiency in EBIS/T are the characteristics of the electron beam (intensity, energy, current density, size), the axial magnetic field and the length of the trap. These devices have the ability to produce very high charge states with a good efficiency [19], but they generally work in pulse operation and have some restrictions with respect to injected beam acceptance (emittance and intensity). Different approaches are considered to improve the characteristics of EBIS/EBIT charge breeders: the improvement of the trapping by injecting the $1+$ ions in a partially compensated electron beam (Coulomb target) [20], the obtaining of a selective containment by applying a radio frequency signal in the ion trap region [21], the modification of the charge state distribution by acting on the intensity and on the energy tuning of the electron beam. This latter may permit to explore charge state distribution manipulation by either breeding ions at a shell closure or by increasing the dielectronic resonance cross-section for a specific ion. In the next two sections significant results and studies are developed.

\section{REX-TRAP/REX-EBIS operation at REX-ISOLDE and HIE project}

At CERN-ISOLDE, the online mass separator facility produces $60 \mathrm{keV} 1+$ ion beams with about twenty five different target materials and three different kinds of ion sources (plasma, surface and laser ion sources). Sixty different elements and at least 600 isotopes have already 
been produced. To produce high energy exotic beams, the REX-ISOLDE facility (Figure 1) permits to accelerate these beams up to a final energy being variable between 0.8 and 3 $\mathrm{MeV} / \mathrm{u}$, the $1+$ beam is injected into the penning trap (REXTRAP) and then charge bred in the REX-EBIS system. Radioactive ions are routinely charge bred in this system [22], some 1+ $\rightarrow \mathrm{n}+$ efficiencies of the whole system (including the REXTRAP one) as a function of the mass over charge ratio are shown Figure 2. The acceptance limitation of the EBIS and its intrinsic pulsed operation, implies the use of a cooler buncher (the penning trap), unfortunately this device has a space charge limitation and rather long cooling times for heavy ions. The charge breeding time to reach the $\mathrm{q}$ charge from the $1+$ delivered by ISOLDE is reported figure 3 . The EBIS by itself has rather short breeding times but the whole process present clear limitations for heavy ions. The main efforts to progress towards the High Intensity and Energy ISOLDE, concerning this system, is to increase the intensity acceptance of the cooler (present limitation $=1.510^{8}$ ions), to decrease its cooling time, and to increase the electron current density and intensity in the EBIS in order to decrease the charge breeding times and to increase the ion beam intensity acceptance.

\section{MAXEBIS for the HITRAP project}

The HITRAP facility is a decelerator for the ions produced at GSI stored in a storage ring (ESR) and extracted at $7 \mathrm{Mev} / \mathrm{u}$. It is composed by a post decelerator (down to $10 \mathrm{q} \mathrm{Kev}$ ), a cooling trap and a precision trap. The MAXEBIS from Frankfurt University has been rebuilt at GSI and will be used to test components of HITRAP, will be a test injector for the cooler trap and will be used to explore the methods permitting to improve EBIS charge breeding (injection optimization, narrow charge state distribution, extracted beam emittance). This setup is now operational, the first results mainly concern the 'accu-mode' (continuous 1+ injection in the EBIS, pulsed extraction) with an Argon 1+ beam injected [16]. 


\section{ECRIS charge breeding activities}

In ECRIS charge breeders the injection of the $1+$ ion beam is performed by the mean of a grounded tube inserted into the plasma chamber, the primary beam is either suddenly decelerated at the extremity of the tube, or is already partly decelerated before the injection like in TRIAC charge breeder [23] or like proposed by TRIUMF [24]. It seems, in fact, that the two solutions are up to now experimentally equivalent with respect to the capture efficiency; when injecting gaseous ions, about $60 \%$ are captured and re-extracted charge bred, for metallic ions the charge bred ions are only $30 \%$ of the $1+$ beam, consequently an effort should be performed in an accurate modelization of the final trajectories of the $1+$ ions at very low energies. The main present studies concern on line experiments with radioactive ions at ISOLDE and experimental studies for the SPIRAL 2 project at LPSC.

\section{The IS397 collaboration at ISOLDE}

Since 2003, The IS397 collaboration [25] performs ECR charge breeding experiments with stable and radioactive ions delivered by ISOLDE, in order to obtain a valuable comparison between EBIS and ECRIS charge breeders. The PHOENIX ECRIS charge breeder has been lent by the CRLC Daresbury and the $\mathrm{n}+$ spectrometer by LPSC Grenoble. About fifteen different isotopes have been studied either in continuous mode (continuous injection and extraction) or in pulsed one, taking benefit of the ability of the ECRIS to trap ions which can be extracted in pulses with the afterglow process [26] (continuous injection and pulsed extraction). Figure 4 shows results obtained in continuous mode, the dispersion of the results may come from the difficulty to measure accurately the $1+$ beam intensity due to the time evolution of the radioactive nuclei number after each proton pulse delivered by the PS- 
Booster from CERN. However, let us notice that these results are not too much different from the ones obtained at LPSC and at TRIUMF and established on dedicated test benches.

The production of molecular compounds in the ISOLDE target source system permits sometimes to avoid the injection of an isobar contaminated RIB into the charge breeder. The ability of the ECRIS charge breeder to break the molecule and then to multi-ionize the radioactive nucleus has been demonstrated for Lanthanum. Other type of contamination may occur when same A/Q beams are issued from the ISOLDE target ion source system, this is the case for ${ }^{48} \mathrm{Ar}^{1+},{ }^{96} \mathrm{Kr}^{2+}$ and ${ }^{144} \mathrm{Xe}^{3+}$ which are simultaneously produced with a FEBIAD source. After injection of these three beams in the ECRIS charge breeder, they are multi-ionized and their charge state distribution depends on the $Z$ of the atom (like in a classical ECR). The shift of each distribution with respect to another, permit, to decrease drastically the contamination of ${ }^{48} \mathrm{Ar}$ when extracting ${ }^{48} \mathrm{Ar}^{9+}$ like measured in [6]. Other physics can be performed with an ECRIS charge breeder at ISOLDE like explained in [27], [28]. Future improvements are planned like explained in the last paragraph of this paper and in [29].

\section{Charge breeder tuning for the SPIRAL2 project $^{b}$}

For the SPIRAL2 facility, which is under construction, the validation of the charge breeding process is performed on the LPSC test bench equipped with the PHOENIX ECRIS charge breeder. The experimental program consists in measuring all the charge breeding characteristics of the beams delivered by the different $1+$ ion sources planned to be used in the target source system. Due to the low intensities of the radioactive ion beams (not directly

\footnotetext{
${ }^{\mathrm{b}}$ This work has been performed in collaboration with GANIL (C. Barué, M. Dubois, G. Gaubert, P. Jardin, N. Lecesne, F. Lemagnen, R. Leroy, M.H. Moscatello, J.Y. Pacquet, A. Savalle, F. Varenne) and funded by the SPIRAL2 project
} 
measurable), the pre-tuning of the accelerator (CIME cyclotron) will be performed on a sufficiently intense stable ion beam with an A/Q similar to the radioactive ions one, then, in order to keep the parameters of the pre-tuning, the magnetic rigidity of the $n+$ line should remain constant when switching from the Stable Beam (SB) to the Radioactive Beam (RB) $[30]$.

Let's recall that in order to capture the $1+$ ion beam into the $n+$ ECR plasma, its final energy must be carefully adjusted $(\Delta \mathrm{V})[31]$ with a precision of about $1 \mathrm{~V}$ for metallic elements and about $10 \mathrm{~V}$ for gaseous ones.

First experiments have been performed to show the feasibility of the accelerator tuning procedure for gaseous type ions, at the LPSC test bench, with the $1+$ MONOBOB source developed by GANIL [32]. Two experiments have been performed, the first one with the couple $\left[\mathrm{Ar}^{7+}(\mathrm{SB}), \mathrm{Kr}^{15+}(\mathrm{RB})\right]$, the second one with $\left[\mathrm{Kr}^{15+}(\mathrm{SB}), \mathrm{Xe}^{23+}(\mathrm{RB})\right]$. Let's detail the first one.

The MONOBOB source is fed with argon, krypton, and helium as support gas, the flow of this latter one is fixed high $\left(4 * 10^{-3} \mathrm{mbar} .1 / \mathrm{s}\right)$ in order to simulate the high pressure existing close to a real target ion source system. The $\mathrm{Ar}^{1+}$ and $\mathrm{Kr}^{1+}$ intensities (at $20 \mathrm{keV}$ ), are then tuned to about a few hundreds nA (not too low, to have a confident and accurate measurement, not too high, to keep the efficiency of the ECR charge breeder). The experiment is performed in two stages, first, a normal $1+/ \mathrm{n}+\left(\mathrm{Kr}^{1+} \rightarrow \mathrm{Kr}^{15+}\right)$ study in order to find the parameters giving an efficient and fast charge breeding of the RB. For the Kr15+ $(\mathrm{A} / \mathrm{Q}=5.594)$, the efficiency yield is found to be $8.7 \%$ and the charge breeding time $80 \mathrm{~ms}$. The parameters of the ECRIS charge breeder are the following: RF frequency $14 \mathrm{GHz}, \mathrm{RF}$ power: $450 \mathrm{~W}$, support gas: Oxygen $-7 \times 10^{-6}$ mbar, $\Delta \mathrm{V}$ : $-80 \mathrm{~V}$. In the second stage of the experiment, the $20 \mathrm{keV} \mathrm{Ar}{ }^{1+}$ beam is selected by the $1+$ spectrometer (bending radius $=0.4 \mathrm{~m}$, deviation angle $=90$ degrees), the magnetic field is thus established at $\mathrm{B}_{\mathrm{Ar}^{1+}}=3217.82$ gauss. 
Then, the charge breeder parameters previously obtained are directly set, the $\mathrm{Ar}^{7+}$ beam $(\mathrm{A} / \mathrm{Q}=5.709)$ is selected by the $\mathrm{n}+$ spectrometer (bending radius $=0.72 \mathrm{~m}$, deviation angle $=$ 120 degrees) with a magnetic field of $\mathrm{B}_{\mathrm{Ar}^{7+}}=674.30$ gauss. With the charge breeder parameters previously set (optima for the production of $\mathrm{Kr}^{15+}$ ), it is recorded that $\mathrm{Ar}^{7+}$ is produced with an efficiency of $1.75 \%$ and a charge breeding time of $30 \mathrm{~ms}$. At this point it is considered that 'the accelerator is pre-tuned with the measurable stable beam', so the magnetic field of the $\mathrm{n}^{+}$spectrometer is not changed any more, the potential of the charge breeder is calculated and set in order to select the $\mathrm{Kr}^{15+}$ and the magnetic field of the $1+$ spectrometer is calculated and set in order to select the $\mathrm{Kr}^{1+}$ for the injection. The respective values are: charge breeder $\mathrm{HV}=20.329 \mathrm{kV}, \mathrm{B}_{\mathrm{Kr}^{1+}}=4710.24$ gauss. It is then recorded that the efficiency yield for a 'blind' tuning of $\mathrm{Kr}^{1+} \rightarrow \mathrm{Kr}^{15+}$ is $7 \%$ and the charge breeding time 80 ms. The two stages of the procedure have been performed in two different days, the tuning of the $1+$ source was then different, the $1+$ beam had an emittance $15 \%$ higher $(7.3 \pi \mathrm{mm}$. mrad RMS) during the second stage, the difference in the efficiency of $1.7 \%$ with respect to the optimum one is certainly due to this different tuning of the $1+$ source. The summary of the experiments is shown Figure 5.

It should be figured out that the measured charge breeding times are much shorter than the ones presented before and than the ones presented by TRIUMF in [24]. The main difference between LPSC and TRIUMF is the support gas nature (Oxygen versus Helium), moreover at LPSC, after one year without venting the charge breeder, it has been noticed that the RF power necessary to have good results was much lower than before. This fact is consistent with the observation of the decrease of oxygen high charge states when injecting a few hundreds nA of heavy $1+$ ions and is a good motivation to build a UHV ECRIS charge breeder. 


\section{EBIS/T-ECRIS comparison}

The charge breeding activities, as seen before, are mainly dedicated to the improvement of these two kind of charge breeders, and finally, to compare their characteristics with respect to emittance, intensity acceptance, charge breeding times and efficiencies. At this moment both have advantages and disadvantages. Light ions are more efficiently bred by EBIS/T, ion beam quality is higher with such devices regarding emittance and superposition of impurities. On the opposite ECR charge breeders have a simple technology which is an important point for a facility dedicated to the production of Radioactive Ion Beams, especially for the maintenance in a radioactive environment. There is no limitation in $1+$ intensity acceptance and it accepts typical $1+$ beam emittances of the presently operational Target Ions Source systems or of the ones under design. Moreover ECRIS charge breeders work in continuous operation like classical ECRIS or can work in pulsed one, taking advantage of its ability to trap ions and to extract them by the afterglow method. The manipulation methods figured out for EBIS don't seem to apply to ECRIS charge breeders unless the ECR community find other processes than frequency increase to narrow charge state distributions.

\section{Future charge breeding activities in Europe}

The 7th Framework Program (FP7 / 2007-2013) is under negotiation, many laboratories have submitted Letters of Intention in the continuity of the FP6 EURONS programme. These activities should focus on the improvement of three characteristics which will impact the future facilities and physics with RIBS: how to improve the purity of the beams delivered, how to increase the efficiency including the charge breeding time and how to get higher charge states?

For the beam purification, the development of a high performance ECRIS with Ultra High Vacuum Technology and optimized support gas injection is considered. The construction of a 
charge over mass energy separator has already shown an impressive effect [33], the improvement of such technique is a key point for the future of efficient charge breeders.

Concerning the improvement of the efficiencies, more sophisticated injection optics simulations should be performed and experimentally tested, the effect of the injection of cooled or bunched beams should be evaluated. For metallic ions, which have lower efficiency yields, it will be interesting and challenging to develop an efficient charge breeder with a hot plasma chamber permitting to prevent too much wall sticking.

Finally, when the ions are captured into the plasma, the way to increase the average charge state is a classical ECRIS challenge. The optimization of the magnetic confinement associated to the classical frequency increase will obviously shift the charge state distribution to higher charges, however technical difficulties may arise to get simultaneously efficient RF power and $1+$ beam injections.

The EBIS charge state breeder development in the framework of FP7 will become part of an Integrated Infrastructure Initiative of "Precision Tools and Atomic Physics Techniques for Fundamental Physics at Accelerators". This JRA is in a preliminary stage of negotiation, the main studies will be on the production techniques for highly charged ions of stable and exotic isotopes, the first time focused on the needs of experiments in traps and storage rings. 
Figure captions:

Figure 1: Schematics of the REX-ISOLDE facility

Figure 2: Charge breeding efficiencies with REXTRAP/REXEBIS at ISOLDE at ISOLDE

Figure 3: Charge breeding times for $1+\rightarrow q+$ in REXEBIS and for the whole process (including cooling time)

Figure 4: Charge breeding efficiencies with PHOENIX at ISOLDE

Figure 5: Experimental procedure for the SPIRAL2 tuning method validation 


\begin{tabular}{|c|c|}
\hline Facility name & Location \\
\hline CYCLONE & UCL- Belgium \\
\hline REX-ISOLDE & CERN switzerland \\
\hline SPIRAL & GANIL France \\
\hline EXCYT & INFN-LNS Sicilia \\
\hline DRIBS & FLNR-JINR, Russia \\
\hline ALTO & IPNO France \\
\hline SISSI & GANIL France \\
\hline IGISOL & U. Jyväskylä Sweden \\
\hline ISOLDE & CERN Switzerland \\
\hline SPIRAL2 & GANIL France \\
\hline HIE-ISOLDE & CERN switzerland \\
\hline SPES & INFN-LNL Italy \\
\hline FAIR & GSI Germany \\
\hline EURISOL & Europe \\
\hline TRIAC & KEK Japan \\
\hline ISAC & TRIUMF Canada \\
\hline RIBLL I-II & IMP, China \\
\hline CARIBU & ANL USA \\
\hline RIBF & VECC, India \\
\hline ISF & NSCL-MSU USA \\
\hline
\end{tabular}

Table 1: Main Radioactive Ion beam facilities and projects 


\section{References}

[1] U. Koster, Eur. Phys. J. A 15, 255 (2002)

[2] B. Harss et al., Rev. Sci. Instrum. 71, 380 (2000)

[3] F. Wenander Proceedings of the XVth International Conference on Electromagnetic Isotope Separators and Techniques Related to their Applications, Deauville - France, June 2007. Charge state breeders: on-line results

[4] Cornell J.C., 17th International Conference on Cyclotrons and their Applications, Tokyo (2004)

[5] H. A. Grunder, Fifth International Conference on Radioactive Nuclear Beams, Divonne, France, 3 April 2000, Nucl. Phys. A 701, 43 (2002)

[6] T. Fritioff et al. Nucl. Instr. Methods Phys. Res. A 556, 31 (2006)

[7] G. Sikler et al. Eur. Phys. J. A 25, s01, 63\{64 (2005)

[8] C. Tamburella, Ph.D. thesis, Universite Denis Diderot PARIS VII, 1996

[9] J.L. Belmont et al. Proceedings of the $13^{\text {th }}$ International Conference on Cyclotrons and their Applications, Vancouver (1993), pp 729-732

[10] B. Visentin et al. Phys. Scr. T71, 204 (1997)

[11] B.H. Wolf et al., Nucl. Instr. Methods Phys. Res. B 204, 428 (2003)

[12] http://www.nupecc.org/

[13] http://www.gsi.de/informationen/jofu/EURONS/index.html

[14] http://www.eurisol.org/site01/index.php

[15] S. Schwarz et al., in these proceedings I8, A high-current EBIT as a charge breeder for the re-acceleration of rare isotopes at the NSCL

[16] O. Kester, in these proceedings PA23, The MAXEBIS at GSI as a test ion source for charge breeding and for HITRAP 
[17] P. Delheij et al., in these proceedings PA21, The EBIT and the TITAN mass spectrometer at TRIUMF-ISAC

[18] E. D. Donets, Rev. Sci. Instrum. 69, 614 (1998)

[19] F. Wenander et al Rev. Sci. Instrum. 77, 03B104 (2006)

[20] R. Becker et al., 9th International Symposium on Electron Beam Ion Sources and Traps, J. Phys., Conference Series 2, 20 (2004)

[21] V. Variale et al, Proc. PAC07, Albuquerque, USA, A18 Radioactive Ions, 1445 (2007)

[22] P. Delahaye et al., Charge Breeding mid term report (Deliverable Task 9 EURISOL)

[23] S.C. Jeong (KEK -Japan) Proceedings of the XVth International Conference on Electromagnetic Isotope Separators and Techniques Related to their Applications, Deauville - France, June 2007. KEKCB-18GHz ECR charge breeder at TRIAC

[24] F. Ames et al., in these proceedings PB29, The ECRIS charge state breeding project at TRIUMF

[25] http://is397-collaboration.web.cern.ch/IS397-collaboration/

[26] P. Delahaye et al., Rev. Sci. Instrum. 77, 03B105 (2006)

[27] M. Marie-Jeanne et al., Proceedings of the XVth International Conference on Electromagnetic Isotope Separators and Techniques Related to their Applications, Deauville - France, June 2007. Charge breeding ions for nuclear physics with the Phoenix ECRIS

[28] M. Marie-Jeanne et al., Proceedings of the International Symposium on Nuclear Astrophysics - Nuclei in the Cosmos - POS(NIC-IX-087) (2006)

[29] P. Delahaye, Proceedings of the XVth International Conference on Electromagnetic Isotope Separators and Techniques Related to their Applications, Deauville - France, June 2007. Potentials of the ECR $1+\rightarrow \mathrm{n}+$ charge breeding for radioactive ions

[30] L. Boy Ph.D. thesis, Universite Pierre et Marie Curie PARIS VI, (1997) 
[31] T. Lamy et al., Rev. Sci. Instrum. 77, 03 B101 (2006)

[32] C. Huet-Equilbec et al., Nucl. Instr. Methods Phys. Res. B 240, 752 (2005)

[33] F. Ames et al., Rev. Sci. Instrum. 77, 03B103 (2006) 